\title{
Clinicopathological characteristics of pancreatic ductal adenocarcinoma with invasive micropapillary carcinoma component with emphasis on the usefulness of PKC $\xi$ immunostaining for detection of reverse polarity
}

\author{
HIRONORI RYOTA $^{1}$, MITSUAKI ISHIDA ${ }^{2}$, YUSUKE EBISU ${ }^{2}$, HIROAKI YANAGIMOTO ${ }^{1}$, \\ TOMOHISA YAMAMOTO $^{1}$, HISASHI KOSAKA ${ }^{1}$, SATOSHI HIROOKA ${ }^{1}$, SO YAMAKI ${ }^{1}$, \\ MASAYA KOTSUKA ${ }^{1}$, YOICHI MATSUI ${ }^{1}$, KOJI TSUTA ${ }^{2}$ and SOHEI SATOI $^{1}$ \\ Departments of ${ }^{1}$ Surgery and ${ }^{2}$ Pathology and Laboratory Medicine, \\ Kansai Medical University, Hirakata, Osaka 573-1010, Japan
}

Received February 1, 2019; Accepted February 17, 2020

DOI: $10.3892 / \mathrm{ol} .2021 .12786$

\begin{abstract}
Invasive micropapillary carcinoma (IMPC) is a rare distinct histopathological subtype, characterized by the presence of carcinoma cells displaying reverse polarity. Only limited clinicopathological information is available regarding pancreatic IMPC. The aim of the present study was to clarify the clinicopathological features of pancreatic IMPC and the usefulness of protein kinase $\mathrm{C}$ (PKC) $\zeta$ immunostaining for the detection of reverse polarity. We reviewed 242 consecutive surgically resected specimens of pancreatic ductal adenocarcinoma and selected samples with an IMPC component. Clinicopathological characteristics were compared between the IMPC and non-IMPC groups. Immunohistochemical staining for $\mathrm{PKC} \zeta$ was performed using an autostainer. In total, 14 cases had an IMPC component (5.8\%). The extent of IMPC component ranged from 5 to $20 \%$. There were no significant differences in tumor location, $\mathrm{T}$ category, lymph node metastatic status, preoperative carbohydrate antigen 19-9 level, resection status and overall survival between the IMPC and non-IMPC groups. Immunostaining for $\mathrm{PKC} \xi$ clearly showed reverse polarity of the neoplastic cells of IMPC. Although previous reports have shown that the presence of an IMPC component ( $>20 \%$ of the tumor) indicated poor prognosis, the
\end{abstract}

Correspondence to: Dr Mitsuaki Ishida, Department of Pathology and Laboratory Medicine, Kansai Medical University, 2-5-1 Shinmachi, Hirakata, Osaka 573-1010, Japan

E-mail: ishidamt@hirakata.kmu.ac.jp

Abbreviations: IMPC, invasive micropapillary carcinoma; EMA, epithelial membrane antigen; MUC1, mucin core protein; PKC, protein kinase C; CA19-9, carbohydrate antigen 19-9

Key words: IMPC, ductal adenocarcinoma, $\mathrm{PKC} \zeta$, immunostaining, pancreatic cancer present study demonstrated that presence of IMPC $<20 \%$ did not suggest a worse prognosis.

\section{Introduction}

Invasive micropapillary carcinoma (IMPC), a rare distinct histopathological subtype, is characterized histopathologically by the presence of small morula-like clusters of carcinoma cells displaying reverse polarity (so-called inside-out pattern), wherein the apical pole of the neoplastic cells faces the stroma side surrounded by clear stromal space (1). This rare variant was originally described in the breast in 1980 (2), and has been increasingly recognized as a distinct histopathological subtype in a variety of organs, including the salivary gland (3), lung (4), stomach (5), colon (5), and urinary bladder (3-6). Recognition of this variant is important because previous reports demonstrated that IMPC shows significantly more frequent lymphovascular invasion and lymph node metastasis (1,3-7). Although rare, IMPC in the pancreas has been described $(8,9)$, but only one case series of IMPC of the pancreas has been reported (8). Khayyata et al analyzed IMPC occurring in the ampullo-pancreatobiliary region, and revealed that $2.6 \%$ of pancreatic carcinomas have an IMPC component $(>20 \%$ of the tumor) and these patients show a poor prognosis (8). However, only limited information regarding the clinicopathological features of IMPC is available.

In order to differentiate IMPC from carcinoma with retraction artifacts, several markers, such as epithelial membrane antigen (EMA), mucin core protein 1 (MUC1), and sialyl-Lewis $\mathrm{X}$, have been suggested for identifying reverse polarity in the neoplastic nests of IMPC (10-12). MUC1 is a type I transmembrane glycoprotein that belongs to the family of mucin proteins and is expressed in various epithelial cells, EMA is also expressed in various epithelial cells. The protein kinase $\mathrm{C}$ (PKC) isoforms are protein kinases involved in multiple signal transduction cascades (13). In particular, the $\mathrm{PKC} \zeta$ isoform is reported that it is required for growth, invasion and tumorigenesis (14), and plays an important role in 
the maintenance of cell polarity and is expressed in the apical portion of epithelial cells $(13,15)$. However, there are no studies using $\mathrm{PKC} \zeta$ immunostaining to detect reverse polarity in the neoplastic nests of IMPC.

The aim of the present study is to clarify the clinicopathological features of IMPC of the pancreas. Moreover, we examine the usefulness of PKC $\zeta$ immunostaining to detect reverse polarity in IMPC.

\section{Materials and methods}

Patients and samples. Two hundred fifty-five patients with pancreatic carcinoma underwent surgery at the Department of Surgery of Kansai Medical University (Osaka, Japan) between January 2006 and December 2015. Patients who had metastasis to other organs, those who died of other causes or complications, and those who were macroscopic surgical margin-positive were excluded. Finally, 242 patients were included in this study.

All resected surgical specimens were reviewed by at least two diagnostic pathologists. We selected patients with ductal adenocarcinoma with an IMPC component and evaluated the extent of IMPC component in the tumor. All patients were staged according to the 8th Union for International Cancer Control TNM Classification (16).

The study was conducted in accordance with the Declaration of Helsinki, and the study protocol was approved by the institutional review board of our hospital (protocol no. 2017272). Informed and written consents were obtained from the patients and their relatives for the use of these clinical materials for research.

Immunohistochemistry. Formalin-fixed and paraffinembedded blocks of the resected specimens were cut into 4- $\mu \mathrm{m}$-thick sections, deparaffinized, and rehydrated. Immunohistochemical analyses were performed using an autostainer (Discovery XT System; Roche Diagnostics) according to the manufacturer's instructions. A mouse monoclonal antibody against MUC1 (H23, prediluted, Roche,), a mouse monoclonal antibody against EMA (E-29, prediluted; Agilent Technologies, Inc.), and a rabbit polyclonal antibody against PKC $\zeta$ (ab59364, 1:30; Abcam plc) were used as primary antibodies. Normal pancreatic ducts were used as a positive control. Immunohistochemical stainings were assessed independently by pathologists blinded to the patients' clinical features.

Statistical analysis. Statistical analyses were performed using JMP 12 software (SAS Institute). Student's t-test was used to analyze continuous variables, and $\chi^{2}$ and Fisher's exact probability tests were used to analyze categorical variables. $\mathrm{P}<0.05$ was considered to indicate a statistically significant difference. Cumulative survival rates were calculated using the Kaplan-Meier method. Statistical differences in survival were calculated using the log-rank test.

\section{Results}

Clinical characteristics. Table I summarizes the clinicopathological features of patients in the present study. This study included 101 women and 141 men. Fourteen patients had an IMPC component (5.8\%). In the IMPC group, 12 patients $(85.7 \%)$ had lymph node metastasis. There were no significant differences in tumor location, $\mathrm{T}$ category, lymph node metastatic status, preoperative serum carbohydrate antigen 19-9 (CA19-9) level, or resection status between the IMPC and non-IMPC groups. Tumor size in the IMPC group ranged from 20 to $65 \mathrm{~mm}$ (mean $37.3 \mathrm{~mm}$; median $38.0 \mathrm{~mm}$ ), and there was no significant difference compared to the non-IMPC group (mean $35.5 \mathrm{~mm}$; median $33.0 \mathrm{~mm}$ ) $(\mathrm{P}=0.69)$.

Histopathological characteristics. The characteristic histopathological features of ductal adenocarcinoma and IMPC are shown in Fig. 1A and B. The neoplastic cells showed a typical inside-out pattern surrounded by clear stromal space. Table II summarizes the clinicopathological characteristics of patients with pancreatic ductal adenocarcinoma with IMPC component. The extent of IMPC component ranged from 5 to $20 \%$ (Table II). IMPC component accounted for $5 \%$ of the tumor in 10 patients, $10 \%$ in 2 patients, and $20 \%$ in 2 patients. All patients had lymphovascular and perineural invasion, and the IMPC component was usually present at the edge of the tumor. Neutrophilic infiltration of the IMPC component was noted in 12 patients $(85.7 \%)$, and abundant neutrophilic infiltration was observed in 7 patients (50\%). Neutrophils were present around and within the tumor nests in the IMPC component (Fig. 1C). In most of these patients, neutrophilic infiltration was much less frequent in the conventional adenocarcinoma region. No neutrophilic infiltration was noted in 2 patients (cases 1 and 13).

Immunohistochemical findings. PKC $\zeta$ was expressed in the apical pole of the neoplastic cells in conventional ductal adenocarcinoma (Fig. 2A). PKC $\zeta$ was detected in the stroma-facing surface of the neoplastic cell clusters of IMPC in all patients with IMPC component, indicating reverse polarity (Fig. 2B). Detection of reverse polarity by $\mathrm{PKC} \zeta$ was clearer than detection by MUC1 or EMA (Fig. 2E and F). As reference, MUC1 and EMA were expressed in the apical pole of the neoplastic cells in conventional ductal adenocarcinoma (Fig. 2C and D).

Clinical outcome. The follow-up period ranged from 5 to 75 months for 14 patients with IMPC component. Six patients $(42.9 \%)$ are still alive (14-75 months). The Kaplan-Meier curve showed no significant difference in overall survival between the IMPC and non-IMPC groups $(\mathrm{P}=0.38)$ (Fig. 3).

\section{Discussion}

In the present study, we demonstrated that the frequency of IMPC in pancreatic ductal adenocarcinoma patients is $5.8 \%$, although the extent of the IMPC component was less than $20 \%$ in all of the patients with IMPC component, and the presence of IMPC component (less than 20\%) did not indicate a worse prognosis. Moreover, we also clearly showed that PKC $\zeta$ is a useful immunohistochemical marker for detecting reverse polarity of IMPC and this is the first report for usefulness. 
Table I. Clinicopathological features of pancreatic invasive ductal adenocarcinoma with and without IMPC component.

\begin{tabular}{|c|c|c|c|}
\hline Variables & IMPC group (\%) & No IMPC group (\%) & P-value \\
\hline Sex & & & 0.0964 \\
\hline Male & $5(35.71)$ & $136(59.65)$ & \\
\hline Female & $9(64.29)$ & $92(40.35)$ & \\
\hline Age, years & & & 0.5183 \\
\hline Mean & 66.50 & 68.19 & \\
\hline Range & $53-82$ & $36-86$ & \\
\hline Location & & & 0.7755 \\
\hline $\mathrm{Ph}$ & $10(71.43)$ & $147(64.47)$ & \\
\hline $\mathrm{Pbt}$ & $4(28.57)$ & $81(35.53)$ & \\
\hline $\mathrm{T}$ category & & & 1.0000 \\
\hline $1+2$ & $9(64.29)$ & $146(64.04)$ & \\
\hline $3+4$ & $5(35.71)$ & $82(35.96)$ & \\
\hline Tumor size, mm & & & 0.6858 \\
\hline Mean & 37.07 & 35.53 & \\
\hline Range & $20-65$ & $9-100$ & \\
\hline Lymph node metastasis & & & 0.3618 \\
\hline Negative & $2(14.29)$ & $64(28.07)$ & \\
\hline Positive & $12(85.71)$ & $164(71.93)$ & \\
\hline $\mathrm{R} 0 / 1$ & & & 0.1879 \\
\hline 0 & $9(64.29)$ & $181(79.39)$ & \\
\hline 1 & $5(35.71)$ & $47(20.61)$ & \\
\hline Serum CA19-9, U/ml & & & 0.5447 \\
\hline$<37$ & $5(35.71)$ & $63(27.63)$ & \\
\hline $37<$ & $9(64.29)$ & $165(72.97)$ & \\
\hline
\end{tabular}

IMPC, invasive micropapillary carcinoma; $\mathrm{Ph}$, pancreatic head; Pbt, pancreatic body and/or tail.
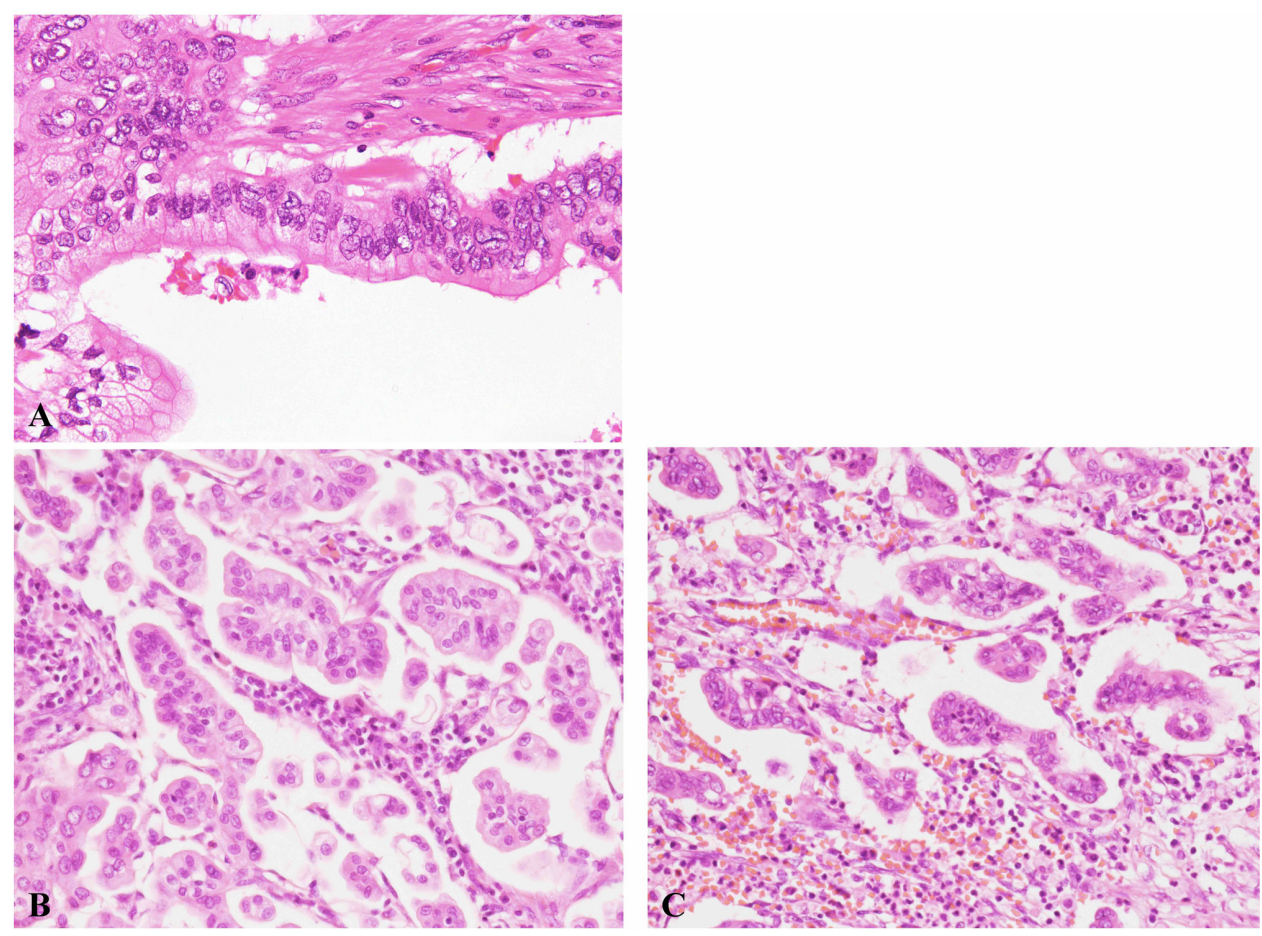

Figure 1. Ductal adenocarcinoma and invasive micropapillary carcinoma. H\&E staining. Magnification, x400. (A) Typical histological features of ductal adenocarcinoma. (B) Typical histological features of invasive micropapillary carcinoma. (C) Neutrons were present around and within the tumor nests. 
Table II. Cases of IMPC component.

\begin{tabular}{cccccccccc}
\hline Case & Sex & Age & $\begin{array}{c}\text { IMPC } \\
\text { component, } \%\end{array}$ & $\begin{array}{c}\text { Tumor } \\
\text { size, } m \text { m }\end{array}$ & N0/1 & ly or v & R0/1 & $\begin{array}{c}\text { Overall survival, } \\
\text { months }\end{array}$ & Dead/Alive \\
\hline 1 & M & 74 & 5 & 45 & 1 & 1 & 1 & 5 & Dead \\
2 & F & 63 & 5 & 20 & 1 & 1 & 0 & 65 & Alive \\
3 & F & 66 & 5 & 35 & 1 & 1 & 1 & 62 & Dead \\
4 & F & 71 & 5 & 38 & 1 & 1 & 1 & 36 & Alive \\
5 & M & 65 & 5 & 24 & 1 & 1 & 0 & 30 & Alive \\
6 & F & 65 & 5 & 30 & 1 & 1 & 1 & 25 & Dead \\
7 & F & 70 & 5 & 40 & 1 & 1 & 0 & 7 & Dead \\
8 & F & 72 & 5 & 45 & 1 & 1 & 0 & 8 & Dead \\
9 & M & 63 & 5 & 42 & 1 & 1 & 0 & 75 & Alive \\
10 & F & 53 & 5 & 30 & 1 & 1 & 0 & 54 & Alive \\
11 & F & 82 & 10 & 65 & 0 & 1 & 0 & 14 & Alive \\
12 & M & 56 & 10 & 32 & 0 & 1 & 0 & 39 & Dead \\
13 & M & 64 & 20 & 38 & 1 & 1 & 0 & 26 & Dead \\
14 & F & 67 & 20 & 35 & 1 & 1 & 1 & 7 & Dead \\
\hline
\end{tabular}

IMPC, invasive micropapillary carcinoma; M, male; F, female.

The clinicopathological significance of IMPC has been reported in several organs, including the breast $(1,7)$ and urinary bladder (6). However, probably due to lack of recognition of this entity in the pancreas, only one case series of IMPC (8) and one case report of pure IMPC (9) in the pancreas have been described in the English literature. Khayyata et al reported IMPC in the pancreas for the first time (8). They defined IMPC as invasive micropapillary architecture in more than $20 \%$ of the tumor, and the incidence of pancreatic IMPC was $2.6 \%$. All IMPC components were located in the pancreas head, and the characteristic histopathological finding was abundant infiltration of neutrophils around the tumor nests of IMPC. In the present series, we examined pancreatic ductal adenocarcinoma with IMPC component regardless of the extent, because the clinicopathological features of pancreatic ductal adenocarcinoma with less than $20 \%$ IMPC component have not been clarified. The frequency of pancreatic IMPC $(5.8 \%)$ in this study was higher than the results of the previous report $(2.6 \%)(8)$, although we included cases where the extent of IMPC component was less than $20 \%$. Neutrophilic infiltration was observed in $85.7 \%$ of patients in the present series, which may be a characteristic feature of pancreatic IMPCs, because this has rarely been described in IMPCs occurring in other organs (1,3-7). Moreover, this study revealed that pancreatic IMPC can develop in the pancreas body and tail (4/14 patients).

Most IMPCs found in various organs have a conventional carcinoma component, and pure IMPC is rare. Even in the breast, where IMPC most frequently develops, pure IMPC accounts for approximately $0.9-2 \%$ of carcinomas $(1,17)$. The amount of micropapillary carcinoma component required for diagnosis of IMPC has not been established yet, although the clinical significance of the extent of IMPC component has been discussed (1). Chen et al analyzed the association of the amount of IMPC component and the clinicopathological parameters, and they concluded that the presence of IMPC component suggested higher incidence of lymph node metastasis. However, there was no significant association between the amount of IMPC component and lymph node and distant metastases, although a trend was noted (18). Khayyata et al reported that the prognosis of pancreatic IMPC (all patients had $>20 \%$ IMPC component) was poor, similar to that of poorly differentiated pancreatic adenocarcinoma (8). In the present series, there was no significant difference in overall survival between the IMPC and non-IMPC groups $(\mathrm{P}=0.38)$. The results of the previous report (8) and the present study do not correspond to the above-mentioned clinical significance of the presence of IMPC component in the breast (18). In this study, the limitation is that the number of target patients is small. Therefore, additional large studies are needed to clarify the clinical significance of the amount of IMPC component in pancreatic ductal adenocarcinoma.

The PKC family is composed of 12 members, and is grouped into three subclasses: Classical, novel, and atypical (13). PKC $\zeta$ is an atypical PKC. Atypical PKCs play an important role in maintaining cell polarity because they are present in the tight junctions, which physically separate the apical and basolateral portions of the cell (13). PKC $\zeta$ has also been reported to play a role in the reorientation of polarity and lumen formation (inside-out pattern) in Madin-Darby canine kidney cells (18). Accordingly, we speculated that PKC $\zeta$ could be used as a marker of reverse polarity in IMPC. We demonstrated that PKC $\zeta$ was expressed in the apical pole of the neoplastic cells in conventional adenocarcinoma; in contrast, its expression was noted in the stroma-facing surface of the neoplastic cell clusters in the IMPC component, clearly showing reverse polarity in IMPC. Several markers for demonstrating reverse polarity in IMPC, including EMA, MUC1, and sialyl-Lewis $\mathrm{X}$, have been reported $(10,11)$. However, immunostaining and evaluation of these markers is 

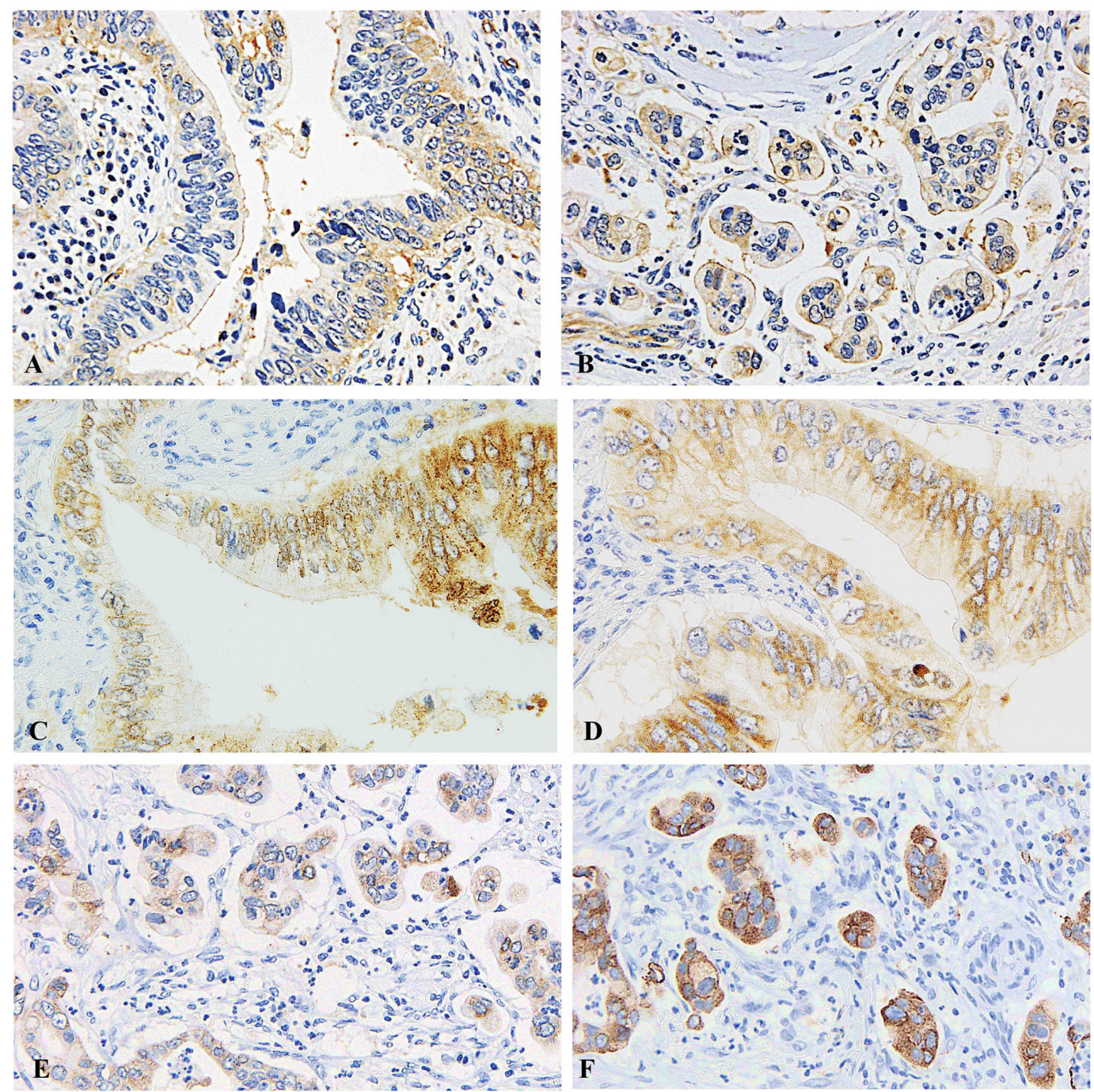

Figure 2. Predominant PKC $\zeta$ immunostaining. Magnification, $\mathrm{x} 400$. (A) On insides of ductal adenocarcinoma. (B) In the stroma facing surface of the cell clusters. (C) Staining with MUC1 on insides of ductal adenocarcinoma. (D) Staining with EMA on insides of ductal adenocarcinoma. (E) Staining with MUC1 on insides of invasive micropapillary carcinoma. (F) Staining with EMA on insides of invasive micropapillary carcinoma. PKC $\zeta$, protein kinase $\mathrm{C} \zeta$; MUC1, Mucin1; EMA, epithelial membrane antigen.

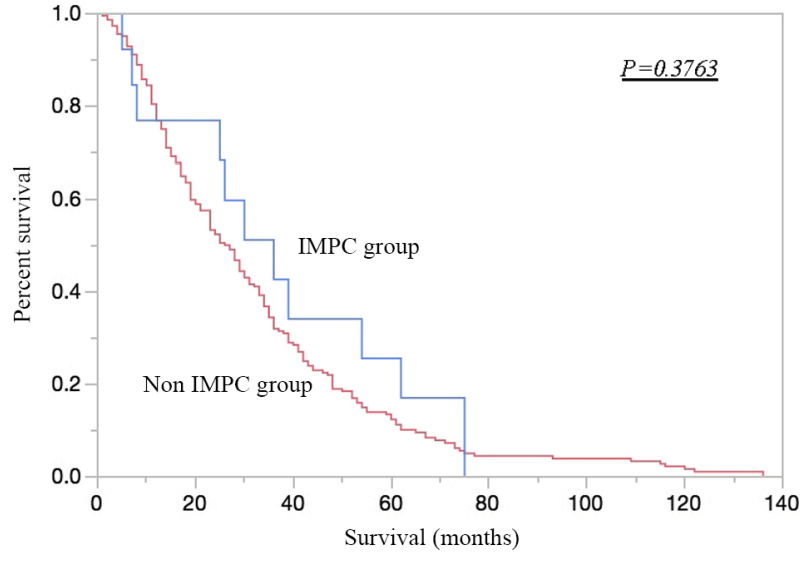

Figure 3. Kaplan-Meier curve for overall survival in the IMPC and non-IMPC groups. IMPC, invasive micropapillary carcinoma.

not always easy due to the background cytoplasmic staining, as shown in Fig. 2E and F. Staining with PKC $\zeta$ was clearer than with EMA and MUC1, and reverse polarity was easier to determine in all cases. The present study demonstrated the usefulness of $\mathrm{PKC} \xi$, an apical marker, to detect reverse polarity in IMPC; therefore, $\mathrm{PKC} \zeta$ must be added as an immunohistochemical marker for detecting reverse polarity, and this marker may be available for IMPC occurring in other organs.

In summary, the present study demonstrated that IMPC component (less than 20\%) did not suggest a worse prognosis $(\mathrm{P}=0.38)$ and there were no significant differences in tumor location, $\mathrm{T}$ category, lymph node metastatic status, preoperative CA19-9 level, or resection status between the IMPC and non-IMPC groups. Moreover, immunostaining for $\mathrm{PKC} \xi$ is useful for demonstrating reverse polarity of neoplastic cells of IMPC.

\section{Acknowledgements}

This abstract was presented at The 50th Annual Congress of the Korean Association of HBP Surgery on April 4, 2019 in Seoul, 
South Korea, and was published as Abstract no. BP OP 2-6. The authors would like to thank Dr Tatsuma Sakaguchi (Department of Surgery, Kansai Medical University, Osaka, Japan) for conference presentation.

\section{Funding}

No funding was received.

\section{Availability of data and materials}

All data generated or analyzed during this study are included in this published article.

\section{Authors' contributions}

HR and MI were responsible for the conception and design of the study. HR and YE performed immunohistochemical analyses. HR, MI, YE, HY, TY, HK, SH, SY, MK, YM, KT and $\mathrm{SS}$ were involved in the acquisition, analysis and interpretation of data. HR and MI confirm the authenticity of all raw data. HR and MI drafted the manuscript and prepared figures. All authors read and approved the final manuscript.

\section{Ethics approval and consent to participate}

The present study was conducted in accordance with the Declaration of Helsinki, and the study protocol was approved by the Kansai Medical University Hospital Ethics Review Committee (protocol no. 2017272). Informed and written consents were obtained from the patients and their relatives for the use of these clinical materials for research.

\section{Patient consent for publication}

Not applicable.

\section{Competing interests}

The authors declare that they have no competing interests.

\section{References}

1. Yang YL, Liu BB, Zhang X and Fu L: Invasive micropapillary carcinoma of the breast: An update. Arch Pathol Lab Med 140: 799-805, 2016

2. Fisher ER, Palekar AS, Redmond C, Barton B and Fisher B: Pathologic findings from the National surgical adjuvant breast project (protocol no. 4). VI. Invasive papillary cancer. Am J Clin Pathol 73: 313-322, 1980

3. Nagao T, Gaffey TA, Visscher DW, Kay PA, Minato H, Serizawa $\mathrm{H}$ and Lewis JE: Invasive micropapillary salivary duct carcinoma: A distinct histologic variant with biologic significance. Am J Surg Pathol 28: 319-326, 2004.
4. Cakir E, Yilmaz A, Demirag F, Oguztuzun S, Sahin S, Yazici UE and Aydin M: Prognostic significance of micropapillary pattern in lung adenocarcinoma and expression of apoptosis-related markers: Caspase-3, bcl-2, and p53. APMIS 119: 574-580, 2011.

5. Guzinska-Ustymowicz K, Niewiarowska K and Pryczynicz A Invasive micropapillary carcinoma: A distinct type of adenocarcinomas in the gastrointestinal tract. World J Gastroenterol 20: 4597-4606, 2014.

6. Comperat E, Roupret M, Yaxley J, Reynolds J, Varinot J, Ouzaïd I, Cussenot $\mathrm{O}$ and Samaratunga $\mathrm{H}$ : Micropapillary urothelial carcinoma of the urinary bladder: A clinicopathological analysis of 72 cases. Pathology 42: 650-654, 2010.

7. Umeda T, Ishida M, Murata S, Mori T, Kawai Y, Itoi N, Tomida K, Tanaka A, Sakai S, Kitamura M, et al: Immunohistochemical analyses of CD44 variant isoforms in invasive micropapillary carcinoma of the breast: Comparison with a concurrent conventional invasive carcinoma of no special type component. Breast Cancer 23: 869-875, 2016.

8. Khayyata S, Basturk O and Adsay NV: Invasive micropapillary carcinomas of the ampullo-pancreatobiliary region and their association with tumor-infiltrating neutrophils. Mod Pathol 18: 1504-1511, 2005.

9. Kitagawa $H$, Nakamura M, Tani T, Tajima $H$, Nakagawara $H$, Ohnishi I, Takamura H, Kayahara M, Ohta T, Zen Y, et al: A pure invasive micropapillary carcinoma of the pancreatic head: Long disease-free survival after pancreatoduodenectomy and adjuvant chemotherapy with gemcitabine. Pancreas 35: 190-192, 2007.

10. Acs G, Esposito NN, Rakosy Z, Laronga C and Zhang PJ: Invasive ductal carcinomas of the breast showing partial reversed cell polarity are associated with lymphatic tumor spread and may represent part of a spectrum of invasive micropapillary carcinoma. Am J Surg Pathol 34: 1637-1646, 2010.

11. Nassar H, Pansare V, Zhang H, Che M, Sakr W, Ali-Fehmi R, Grignon D, Sarkar F, Cheng J and Adsay V: Pathogenesis of invasive micropapillary carcinoma: Role of MUC1 glycoprotein. Mod Pathol 17: 1045-1050, 2004

12. Wei J, Cui L, Liu F, Fan Y, Lang R, Gu F, Guo X, Tang P and $\mathrm{Fu}$ L: E-selectin and Sialyl Lewis X expression is associated with lymph node metastasis of invasive micropapillary carcinoma of the breast. Int J Surg Pathol 18: 193-200, 2010.

13. Rosse C, Linch M, Kermorgant S, Cameron AJ, Boeckeler K and Parker PJ: PKC and the control of localized signal dynamics. Nat Rev Mol Cell Biol 11: 103-112, 2010.

14. Butler AM, Scotti Buzhardt ML, Li S, Smith KE, Fields AP and Murray NR: Protein kinase $\mathrm{C}$ zeta regulates human pancreatic cancer cell transformed growth and invasion through a STAT3-dependent mechanism. PLoS One 8: e72061, 2013.

15. Whyte J, Thornton L, McNally S, McCarthy S, Lanigan F, Gallagher WM, Stein T and Martin F: PKCzeta regulates cell polarisation and proliferation restriction during mammary acinus formation. J Cell Sci 123: 3316-3328, 2010.

16. Brierley J, Gospodarowicz MK and Wittekind C: TNM classification of malignant tumours. John Wiley \& Sons, Inc., Chichester, Hoboken, NJ, 2017.

17. Guo X, Chen L, Lang R, Fan Y, Zhang X and Fu L: Invasive micropapillary carcinoma of the breast: Association of pathologic features with lymph node metastasis. Am J Clin Pathol 126: 740-746, 2006.

18. Chen L, Fan Y, Lang RG, Guo XJ, Sun YL, Cui LF, Liu FF, Wei J, Zhang XM and Fu L: Breast carcinoma with micropapillary features: Clinicopathologic study and long-term follow-up of 100 cases. Int J Surg Pathol 16: 155-163, 2008.

This work is licensed under a Creative Commons Attribution-NonCommercial-NoDerivatives 4.0 International (CC BY-NC-ND 4.0) License. 